Computer Science and Artificial Intelligence Laboratory Technical Report

MIT-CSAIL-TR-2006-061

September 5, 2006

Refactoring for parameterizing Java classes Adam Kiezun, Michael D. Ernst, Frank Tip, and Robert M. Fuhrer 


\section{Refactoring for Parameterizing Java Classes}

\author{
MIT CS\&AI Lab \\ 32 Vassar Street, Cambridge, MA 02139, USA \\ \{akiezun, mernst $\} @ c s a i l . m i t . e d u$
}

\author{
Frank Tip Robert M. Fuhrer \\ IBM T.J. Watson Research Center \\ P.O. Box 704, Yorktown Heights, NY 10598, USA \\ $\{$ ftip,rfuhrer\}@us.ibm.com
}

\begin{abstract}
Type safety and expressiveness of many existing Java libraries and their client applications would improve, if the libraries were upgraded to define generic classes. Efficient and accurate tools exist to assist client applications to use generics libraries, but so far the libraries themselves must be parameterized manually, which is a tedious, time-consuming, and error-prone task. We present a typeconstraint-based algorithm for converting non-generic libraries to add type parameters. The algorithm handles the full Java language and preserves backward compatibility, thus making it safe for existing clients. Among other features, it is capable of inferring wildcard types and introducing type parameters for mutually-dependent classes. We have implemented the algorithm as a fully automatic refactoring in Eclipse.

We evaluated our work in two ways. First, our tool parameterized code that was lacking type parameters. We contacted the developers of several of these applications, and in all cases where we received a response, they confirmed that the resulting parameterizations were correct and useful. Second, to better quantify its effectiveness, our tool parameterized classes from already-generic libraries, and we compared the results to those that were created by the libraries' authors. Our tool performed the refactoring accurately-in $87 \%$ of cases the results were as good as those created manually by a human expert, in $9 \%$ of cases the tool results were better, and in $4 \%$ of cases the tool results were worse.
\end{abstract}

\section{Introduction}

Generics (a form of parametric polymorphism) are a feature of the Java 1.5 programming language. Generics enable the creation of type-safe reusable classes, which significantly reduces the need for potentially unsafe down-casts in source code. Much pre-1.5 Java code would benefit from being upgraded to use generics. Even new code can benefit, because a common programming methodology is to write non-generic code first and convert it later. The task of introducing generics to existing code can be viewed as two related technical problems [8]:

1. The parameterization problem consists of adding type parameters to an existing class definition so that it can be used in different contexts without the loss of type information. For example, one might convert the class definition class Arraylist $\{\ldots\}$ into class ArrayList $<\mathrm{T}>\{\ldots\}$, with certain uses of Object in the body replaced by $\mathrm{T}$.

2. Once a class has been parameterized, the instantiation problem is the task of determining the type arguments that should be given to instances of the generic class in client code. For example, this might convert a declaration ArrayList names; into ArrayList<String> names; .

The former problem subsumes the latter because the introduction of type parameters often requires the instantiation of generic classes. For example, if class HashSet uses a HashMap as an internal representation of the set, then parameterizing the HashSet class requires instantiating the references to HashMap in the body of HashSet.

If no parameterization is necessary, the instantiation problem can be solved using completely automatic and scalable tech- niques $[8,11]$, and the INFER GENERIC TYPE ARGUMENTS refactoring in Eclipse 3.1 is based on our previous work [11]. However, to our knowledge, no previous practical and satisfactory solution to the parameterization problem exists. Thus far, class libraries such as the Java Collections Framework have been parameterized manually, and developers involved with this task described it as very time-consuming, tedious, and error-prone $[12,2]$.

We present a solution to the parameterization problem such that: (i) the behavior of any client of the parameterized classes is preserved, (ii) the translation produces a result similar to that which would be produced manually by a skilled programmer, and (iii) the approach is practical in that it admits an efficient implementation that is easy to use. Our approach fully supports Java 1.5 generics, including bounded and unbounded wildcards, and it has been implemented as a refactoring in Eclipse. Previous approaches for solving the parameterization problem $[9,7,22]$ did not include a practical implementation, and produced incorrect or suboptimal results, as will be discussed in Section 5 .

We evaluated our work in two ways. First, we parameterized non-generic classes, and examined the results to ensure that they were satisfactory and usable to clients. Second, we complemented that qualitative analysis with a quantitative one in which we compared its results to those produced by human programmers. Our tool computes a solution that is nearly identical to the hand-crafted one, and is sometimes even better (i.e., it permits more casts to be removed).

The remainder of this paper is organized as follows. Section 2 gives a motivating example to illustrate the problem and our solution. Section 3 presents our class parameterization algorithm. Section 4 describes the experiments we performed to evaluate our work. Section 5 overviews related work, and Section 6 concludes.

\section{Example}

Figure 1 shows an example program consisting of two classes, MultiSet and SortSet, before and after automatic parameterization by our tool. The following observations can be made about the refactored source code:

1. On line 6, the type of the parameter of MultiSet.add() has been changed to $\mathrm{T}_{1}$, a new type parameter of class MultiSet that represents the type of its elements.

2. On line 9, the return type of MultiSet.getMostCommon() is now $\mathrm{T}_{1}$. This, in turn, required parameterizing class SortSet with a type parameter $\mathrm{T}_{2}$ (line 34 ) and changing the return type of SortSet.getMostCommon() (line 45) to $\mathrm{T}_{2}$. This illustrates that parameterizing one class may require parameterizing others.

3. On line 12, the parameter of MultiSet.addAll() now has type Collection $<$ ? extends $\mathrm{T}_{1}>$, a bounded wildcard type that allows any Collection that is parameterized with a subtype of the receiver's type argument $\mathrm{T}_{1}$ to be passed as an argument. The use of a wildcard is very important here. Suppose that the type Collection $\left\langle\mathrm{T}_{1}>\right.$ were used instead. Then a (safe) call to addAll() on a receiver of type MultiSet<Number> with an actual parameter of type List<Integer> would not compile; the client would be forbidden from using those (desirable) types. 
$1 / /$ A MultiSet may contain a given element more than once. $2 / /$ Each element is associated with a count (a cardinality). 3 public class Multiset \{

// counts maps each element to its number of occurrences. private Map counts = new HashMap ();

public void add(Object t1) \{ \} counts.put(t1, new Integer(getCount $(t 1)+1))$;

public Object getMostCommon() \{

public void addAll(Collection c1) \{ for (Iterator iter $=$ c1.iterator (); iter.hasNext ()$;)\{$

Figure 1. Classes MultiSet and SortSet before and after parameterization by our tool. In the right column, modified declarations are underlined and a removed cast is struck through. The example uses collection classes from package java.util in the standard Java 1.5 libraries: Map, HashMap, Set, Collection, TreeSet.

4. On line 18, the type of the parameter of MultiSet.contains() remains object. This is desirable and corresponds to the (manual) parameterization of the JDK libraries. Suppose the parameter of contains() had type $\mathrm{T}_{1}$ instead, and consider a client that adds only Integers to a Multiset and that passes an Object to contains() at least once on that MultiSet. Such a client would have to declare the MultiSet suboptimally as MultiSet<Object>, rather than MultiSet<Integer> as permitted by our solution.

5. On line 21, the type of the parameter of MultiSet . containsAll() has become an unbounded wildcard Collection<?> (which is shorthand for Collection<? extends Object>). Analogously with the contains() example above, use of Collection $\left\langle\mathrm{T}_{1}\right\rangle$ would force a less precise parameterization of some instances of Multiset in client code.

6. On line 28, the return type of MultiSet.getAllElements() is parameterized as Set $\left\langle\mathrm{T}_{1}\right\rangle$. It is important not to parameterize it with a wildcard, as that would severely constrain client uses of the method's return value (e.g., it would be illegal to add elements other than null to the returned set.)

7. On line 42, the type of the parameter of method SortSet. addAll () is parameterized as Collection<? extends $\mathrm{T}_{2}>$.
1 // A MultiSet may contain a given element more than once. $2 / /$ Each element is associated with a count (a cardinality). 3 public class MultiSet $\left\langle\mathrm{T}_{1}\right\rangle$ \{

// counts maps each element to its number of occurrences.

private Map $\left\langle\mathrm{T}_{1}\right.$, Integer $>$ counts = new HashMap $<\mathrm{T}_{1}$, Integer $>()$; public void add( $\mathrm{T}_{1}$ t1) \}

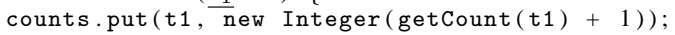

public $\mathrm{T}_{1}$ getMostCommon()

public void addAll(Collection<? extends $\left.\mathrm{T}_{1}>\mathrm{c} 1\right)\{$ for (Iterator $<$ ? extends $\mathrm{T}_{1}>$ iter = c1.iterator (); iter.hasNext (); ) \{

\} add (iter.next ())

public boolean contains(Object o1) \{ \}$^{r}$

public boolean containsall(Collection $\langle$ ? > c2) \{ \} public int getCount(Object o2) \{ return (! contains (o2)) ? 0 : \} ((Integer) counts get (o2)). intValue ();

public Set $\left\langle\mathrm{T}_{1}\right\rangle$ getallElements () \{

Any other parameterization would be incorrect because the method overrides the method TreeSet.addAll(), and the signatures of these methods must remain identical to preserve the overriding relationship [12].

Even for this simple example, the desired parameterization requires 19 non-trivial changes to the program's type annotations, and involves subtle reasoning. In short, class parameterization is a complex process, and automated tool assistance is highly desirable.

Finally, we remark that, although the example uses the standard (generic) Java libraries (e.g., Map $\langle\mathrm{K}, \mathrm{V}\rangle$, Set $\langle\mathrm{E}\rangle$, etc.), our technique is also applicable to classes that do not depend on generic classes.

\section{Algorithm}

Our parameterization algorithm has the following 3 steps:

1. Create type constraints for all program constructs, and add additional constraints using a set of closure rules.

2. Solve the constraints to determine a type for each declaration. 3. Rewrite the program's source code accordingly.

After Section 3.1 presents the notation used for representing type constraints, Sections 3.2-3.4 present the steps of the algorithm, 


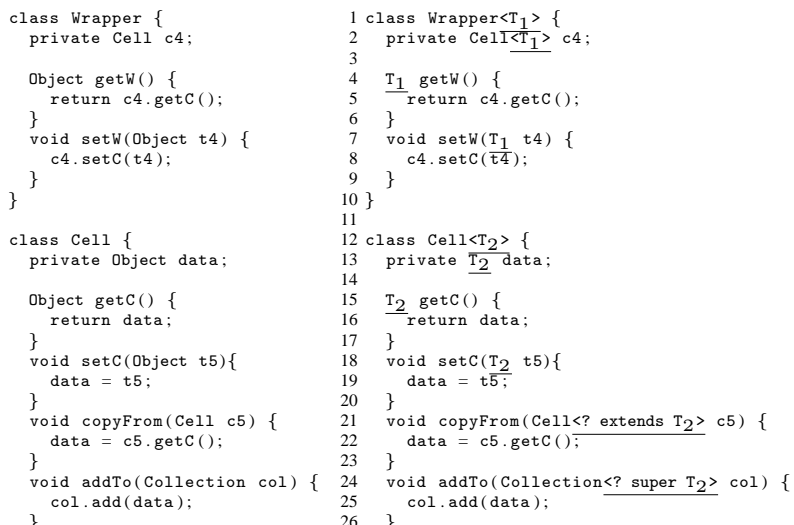

Figure 2. Classes Wrapper and Cell before and after automatic parameterization. In the right column, modified declarations are underlined.

illustrating them with the running example of Figure 2. While Figure 2 is small for the sake of exposition, our algorithm and implementation handle the full Java language. Readers interested primarily in the intuition may skip the detailed treatment of the running example.

\subsection{Type Constraints}

This paper generalizes and extends a framework of type constraints [17] that has been used for refactoring [21, 6, 1] and, in particular, as the basis for a refactoring that solves the instantiation problem [11] (i.e., inferring the type arguments that should be given to generic classes in client code). Due to space limitations, the pre-existing parts of the type constraints formalism are described informally, and the presentation focuses on the new constraints notation and algorithmic contributions that are needed for solving the parameterization problem.

Type constraints are a formalism for expressing subtype relationships between program entities that are required for preserving the type-correctness of program constructs. Consider an assignment $\mathrm{x}=\mathrm{y}$. The constraint $[\mathrm{y}] \leq[\mathrm{x}]$ states that the type of $\mathrm{y}$ (represented by the constraint variable [y]) must be a subtype of the type of $\mathrm{x}$. If the original program is type-correct, this constraint holds. The refactoring must preserve the subtype relationship $[\mathrm{y}] \leq[\mathrm{x}]$ so that the refactored program is type-correct. As another example, consider a method Sub.foo (Object p) that overrides a method Super.foo(Object $q$ ). The refactored program must preserve the overriding relationship, in order to preserve dynamic dispatch behavior. This is guaranteed if the refactored program satisfies a constraint $[\mathrm{p}]=[\mathrm{q}]$ stating that the types of $\mathrm{p}$ and $\mathrm{q}$ are identical.

Our algorithm generates type constraints from a program's abstract syntax tree (AST) in a syntax-directed manner. A solution to the resulting constraint system corresponds to a refactored version of the program for which type-correctness and program behavior is preserved. Frequently, many legal solutions exist, all of which preserve the program's semantics, but some of which are more useful to clients. Our algorithm uses heuristics (Section 3.3.1) to choose among legal solutions, but it never violates the semantics of the program by changing behavior.

Refactoring for parameterization is significantly more complex than previous work because it involves the introduction of formal type parameters with inheritance relations between them, while simultaneously rewriting existing declarations to refer to these new type parameters. This required non-trivial extensions and modifications to the type constraints formalism and the solver, including most notably:

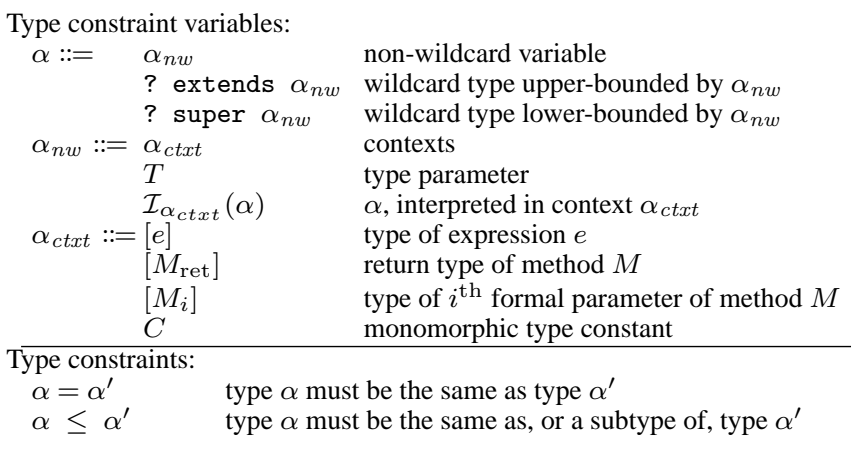

Figure 3. Notation used for defining type constraints.

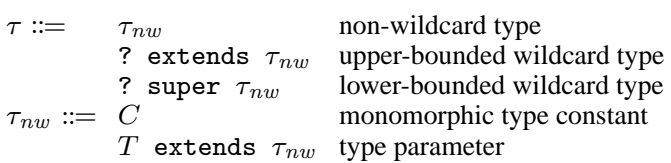

Figure 4. Grammar of types used in the analysis.

1. the introduction of context constraint variables representing the type with which newly introduced type parameters are instantiated,

2. the introduction of wildcard constraint variables to accommodate wildcard types, and

3. the use of heuristics to guide the solver towards solutions preferred by human programmers (e.g., not introducing too many type parameters, and preferring solutions with wildcard types in certain cases), without violating program semantics.

Figure 3 presents the type constraint notation. Type constraints are of the form $\alpha \leq \alpha^{\prime}$ or $\alpha=\alpha^{\prime}$, where $\alpha$ and $\alpha^{\prime}$ are constraint variables. Most forms of constraint variables are standard, but we discuss the new forms context variables and wildcard variables.

Context Variables. This paper introduces a new form of constraint variable that represent the type with which a (newly introduced) formal type parameter is instantiated. Such a context variable is of the form $\mathcal{I}_{\alpha^{\prime}}(\alpha)$ and represents the interpretation of constraint variable $\alpha$ in a context given by constraint variable $\alpha^{\prime}$.

We give the intuition behind context variables using examples.

- Consider the JDK class List<E>. References to its type parameter E only make sense within the definition of List. In the context of an instance of List<String>, the interpretation of $\mathrm{E}$ is String, while in the context of an instance of List<Number $>$, the interpretation of $\mathrm{E}$ is Number. We write $\mathcal{I}_{[\mathrm{x}]}(\mathrm{E})$ for the interpretation of $\mathrm{E}$ in the context of variable $\mathrm{x}$.

- Consider the call c4.setc(t4) on line 8 of Figure 2. Java requires the type of the actual parameter $t 4$ to be a subtype of the formal parameter t5 of Cell.setC (line 18). This is expressed by the constraint $[\mathrm{t} 4] \leq \mathcal{I}_{[\mathrm{c} 4]}(\mathrm{t} 5)$, which means that the type of $\mathrm{t} 4$ is a subtype of the type of $\mathrm{t} 5$, as interpreted by interpretation function $\mathcal{I}_{[\mathrm{c} 4]}$. This interpretation function maps the formal type parameters in the declared type of $c 4$ to the types with which they are instantiated.

Using a context variable here is important. Generating a constraint without a context i.e., $[\mathrm{t} 4] \leq[\mathrm{t} 5]$, would be incorrect because when $[\mathrm{t} 5]$ gets resolved to a type parameter $\mathrm{T}_{2}$, because there is no subtype relationship between the type $T_{1}$ of [t4] and the type $\mathrm{T}_{2}$ of $[\mathrm{t} 5]$.

Although $t 5$ is declared to be of type object on line 18 , it would be incorrect to require that $[\mathrm{t} 4] \leq$ object, because $t 5$ 's type may be rewritten, e.g., to a newly introduced formal type parameter. Our algorithm eventually resolves [t4] to $\mathrm{T}_{1}$ and $[\mathrm{t} 5]$ 


$$
\frac{\text { assignment } e_{1}=e_{2}}{\left\{\left[e_{2}\right] \leq\left[e_{1}\right]\right\}}
$$

statement return $e_{0}$ in method $M$

$$
\left\{\left[e_{0}\right] \leq\left[M_{\text {ret }}\right]\right\}
$$

call $e \equiv e_{0} \cdot m\left(e_{1}, \ldots, e_{k}\right)$ to instance method $M$ canAddParams $\equiv \operatorname{Decl}(M) \in$ TargetClasses

$$
\begin{gathered}
C G e n\left([e],=,\left[M_{\mathrm{ret}}\right],\left[e_{0}\right], \text { canAddParams }\right) \cup \\
\bigcup_{1 \leq i \leq k} C G e n\left(\left[e_{i}\right], \leq,\left[M_{i}\right],\left[e_{0}\right], \text { canAddParams }\right)
\end{gathered}
$$

declaration of method $M, M$ overrides $M^{\prime}$ canAddParams ${ }^{\prime} \equiv \operatorname{Decl}\left(M^{\prime}\right) \in$ TargetClasses

$$
k=\operatorname{NumParams}(M)
$$

$C \overline{G e n W i l d}\left(\left[M_{\text {ret }}\right], \leq,\left[M_{\text {ret }}^{\prime}\right], \operatorname{Decl}(M)\right.$, canAddParams $\left.^{\prime}\right) \cup$

$\bigcup_{1 \leq i \leq k} \operatorname{CGenWild}\left(\left[M_{i}\right],=,\left[M_{i}^{\prime}\right], \operatorname{Decl}(M)\right.$, canAddParams $\left.{ }^{\prime}\right)$

$$
\begin{gathered}
\frac{\alpha_{1} \leq \alpha_{2} \quad \mathcal{I}_{\alpha^{\prime}}\left(\alpha_{1}\right) \text { or } \mathcal{I}_{\alpha^{\prime}}\left(\alpha_{2}\right) \text { exists }}{\mathcal{I}_{\alpha^{\prime}}\left(\alpha_{1}\right) \leq \mathcal{I}_{\alpha^{\prime}}\left(\alpha_{2}\right)} \\
\frac{\alpha_{1} \leq \alpha_{2} \quad \mathcal{I}_{\alpha_{1}}(\alpha) \text { or } \mathcal{I}_{\alpha_{2}}(\alpha) \text { exists }}{\mathcal{I}_{\alpha_{1}}(\alpha)=\mathcal{I}_{\alpha_{2}}(\alpha)} \\
\frac{C\left\langle T_{1}, \ldots, T_{n}\right\rangle \text { extends } / \text { implements } C^{\prime}\left\langle\tau_{1}, \ldots, \tau_{k}\right\rangle}{C G e n\left(C, \leq, C^{\prime}\left\langle\tau_{1}, \ldots, \tau_{k}\right\rangle,{ }_{,}, \text {true }\right)}
\end{gathered}
$$

Figure 5. Representative examples of rules for generating type constraints from Java constructs (rules (r1)-(r4)) and of closure rules (rules (r7)-(r8)). Figure 6 shows auxiliary definitions used by the rules. TargetClasses is a set of classes that should be parameterized by adding type parameters. $\operatorname{Decl}(M)$ denotes the class that declares method $M$.

to $\mathrm{T}_{2}$, implying that $\mathcal{I}_{[\mathrm{c} 4]}$ maps $\mathrm{T}_{2}$ to $\mathrm{T}_{1}$, and thus that $\mathcal{I}_{[\mathrm{c} 4]}(\mathrm{t} 5)$ is resolved to $\mathrm{T}_{1}$.

- In some cases, a context $\alpha_{c t x t}$ is irrelevant. For example, $\mathcal{I}_{\alpha_{c t x t}}$ (String) always resolves to String, regardless of the context $\alpha_{c t x t}$ in which it is interpreted.

Wildcard Variables. There are two situations where our algorithm introduces wildcards in the refactored program.

Wildcard variables are of the form ? extends $\alpha$ or ? super $\alpha$ (where $\alpha$ is another constraint variable), and are used in cases where Java's typing rules require the use of wildcard types. For example, in Figure 1, SortSet.addAll() (line 42) overrides java.util. TreeSet.addAll(). If SortSet becomes a generic class with formal type parameter $\mathrm{T}_{2}$, then preserving this overriding relationship requires the formal parameter $\mathrm{c} 3$ of SortSet.addAll() to have the same type as that of TreeSet.addAll (), which is declared in the Java standard libraries as TreeSet.addAll(Collection<? extends E>). Three parts of our algorithm work together to accomplish this: (i) The type of $c 3$ is represented, using a context variable, as Collection $\left\langle\mathcal{I}_{[\mathrm{c} 3]}(E)\right\rangle$, (ii) Type constraint generation (Section 3.2) produces $\mathcal{I}_{[\mathrm{c} 3]}(E)=$ ? extends $\mathcal{I}_{\text {SortSet }}(E)$, which uses a wildcard variable, and (iii) Constraint solving (Section 3.3) resolves $\mathcal{I}_{\text {SortSet }}(E)$ to $\mathrm{T}_{2}$.

Our algorithm also introduces wildcard types in cases where that results in a more flexible solution, as discussed in Section 3.3.1. However, this does not involve the use of wildcard variables.

\subsection{Type Constraint Generation}

Figure 5 shows a few representative rules for generating type constraints. The rules omitted from Figure 5 involve no significantly different analysis.

Rules (r1) and (r2) are from previous work. Rule (r1) states that the type of the right-hand side of an assignment must be equal to or a subtype of the left-hand side. Rule (r2) states that if a method contains a statement "return $e_{0}$ ", then the type of the returned expression $e_{0}$ must be equal to or a subtype of the method's declared return type. The complete set of rules $[21,11]$ is omitted for brevity and covers the entire Java language.

Rules (r3) and (r4) are among the new rules introduced in this research. Rules (r3) and (r4) govern method calls. Rule (r3) states that the type of the method call expression is the same as the return type of the method (in the context of the receiver). This corresponds to how the type checker treats a method call (i.e., the type of the call and the type of the method are the same). Rule (r4) relates the actual and formal type parameters of the call. The TargetClasses set (a user input to the algorithm) indicates which classes should be refactored by adding type parameters (e.g., in Figure 1, classes MultiSet, SortSet, and the anonymous class declared on line 35 are assumed to be in TargetClasses). The auxiliary function CGen, defined in Figure 6, performs the actual generation of a set of constraints.

Java's type rules impose certain restrictions on parametric types. Closure rules such as (r7) and (r8) in Figure 5 enforce those restrictions. Rule ( $r 7)$ requires that, given two formal type parameters ${ }^{1}$ $T_{1}$ and $T_{2}$ such that $T_{1} \leq T_{2}$ and any context $\alpha$ in which either actual type parameter $\mathcal{I}_{\alpha}\left(T_{1}\right)$ or $\mathcal{I}_{\alpha}\left(T_{2}\right)$ exists, the subtyping relationship $\mathcal{I}_{\alpha}\left(T_{1}\right) \leq \mathcal{I}_{\alpha}\left(T_{2}\right)$ must also hold. To illustrate this rule, consider a class $\mathrm{C}<\mathrm{T} 1$, T2 extends $\mathrm{T} 1>$ and any instantiation $\mathrm{C}<\mathrm{C} 1, \mathrm{C} 2>$. Then, $\mathrm{C} 2 \leq \mathrm{C} 1$ must hold, implying that e.g., $\mathrm{C}<$ Number, Integer $>$ is legal but that $\mathrm{C}<$ Integer, Number $>$ is not. Rule (r8) enforces invariant subtyping ${ }^{2}$ of parametric types: $C\langle\tau\rangle$ is a subtype of $C\left\langle\tau^{\prime}\right\rangle$ iff $\tau=\tau^{\prime}$.

Examples. The following examples show how the rules of Figure 5 apply to three program constructs in the example of Figure 2. The examples assume that the set TargetClasses is $\{$ Cell,Wrapper $\}$.

line 5: call $c 4 \cdot \operatorname{getc}()$ to method $\operatorname{Cell} \cdot \operatorname{getC}()$

$\begin{array}{ll}\stackrel{(\mathrm{r3})}{\longrightarrow} & C G e n\left([\mathrm{c} 4 \cdot \operatorname{getC}()],=,\left[\mathrm{Cell} \cdot \operatorname{get} \mathrm{C}_{\mathrm{ret}}\right],[\mathrm{c} 4], \text { true }\right) \\ \stackrel{(\mathrm{c})}{\rightarrow} & \left\{[\mathrm{c} 4 \cdot \operatorname{getC}()]=\mathcal{I}_{[\mathrm{c} 4]}\left(\left[\mathrm{Cell} \cdot \operatorname{get} \mathrm{C}_{\mathrm{ret}}\right]\right)\right\}\end{array}$

This constraint expresses that the type of the expression [c4.getC()] is the same as the return type of method Cell.getC in the context of the receiver $c 4$.

line 8: call $c 4 . \operatorname{setC}(t 4)$ to method Cell. $\operatorname{setC}($ Object)

$\stackrel{(\mathrm{r} 4)}{\longrightarrow} \quad C G e n\left([\mathrm{t} 4], \leq,\left[\mathrm{Cell} . \mathrm{set}_{1}\right],[\mathrm{c} 4]\right.$, true $)$

$\stackrel{(\mathrm{c} 2)}{\longrightarrow} \quad\left\{[\mathrm{t} 4] \leq \mathcal{I}_{[\mathrm{c} 4]}\left(\left[\mathrm{Cell} . \operatorname{setC}_{1}\right]\right)\right\}$

In other words, the type of $t 4$ must be a subtype of the type of the first parameter of Cell.setC in the context of the type of c4. This constraint is shown as (x2) in Figure 7. (Cell. setC $\mathrm{C}_{1}$ and $t 5$ denote the same program element.)

line 25: call col add (data) to method $M \equiv$ Collection $\langle E\rangle$. add(E)

$\stackrel{(\mathrm{r} 4)}{\rightarrow} \quad C G e n\left([\right.$ data $], \leq,\left[M_{1}\right],[\mathrm{col}]$, false $)$

$\stackrel{(\mathrm{c} 4)}{\rightarrow} \quad\left\{[\right.$ data $\left.] \leq \mathcal{I}_{[\mathrm{col}]}(E)\right\}$

This indicates that data's type must be a subtype of type parameter $\mathrm{E}$ in the context of col. This constraint is shown as (x6) in Figure 7.

Figure 7 (rows (x1)-(x6)) summarizes the constraints generated for Figure 2. For simplicity of presentation, Figure 7 shows the constraint system after eliminating equality constraints. For example, line 5 gives rise to 2 constraints:

(i) $[\mathrm{c} 4 \cdot \operatorname{getC}()] \leq\left[\right.$ Wrapper.get $\left.\mathrm{W}_{\text {ret }}\right] \quad$ rule (r2)

(ii) $[\mathrm{c} 4 \cdot \operatorname{getC}()]=\mathcal{I}_{[\mathrm{c} 4]}\left(\left[\mathrm{Cell} \cdot \operatorname{get} \mathrm{Cret}_{\mathrm{ret}}\right]\right) \quad$ rule $(\mathrm{r} 3)$

\footnotetext{
${ }^{1}$ or constraint variables that could become formal type parameters

${ }^{2}$ In the presence of wildcard types, this is relaxed to 'containment' subtyping [12] (e.g., ? extends Number is contained in ? extends Object and therefore Set<? extends Number $\rangle$ is subtype of Set $\langle$ ? extends Object $\rangle$ ). In this paper and in our implementation, we conservatively assume invariant subtyping even with wildcard types.
} 
$\alpha_{\mathcal{P}}$ is the type, in the original program $\mathcal{P}$, of the program construct corresponding to $\alpha$.

$C G e n$ creates and returns a set of type constraints. The result constrains $\alpha$ and the interpretation of $\alpha^{\prime}$ in context $\alpha^{\prime \prime}$. The particular constraint (=, $\leq$, or $\geq$ ) between $\alpha$ and $\mathcal{I}_{\alpha^{\prime \prime}}\left(\alpha^{\prime}\right)$ is determined by op. CGen is defined by case analysis on the type of its third parameter in the original program $\mathcal{P}$.

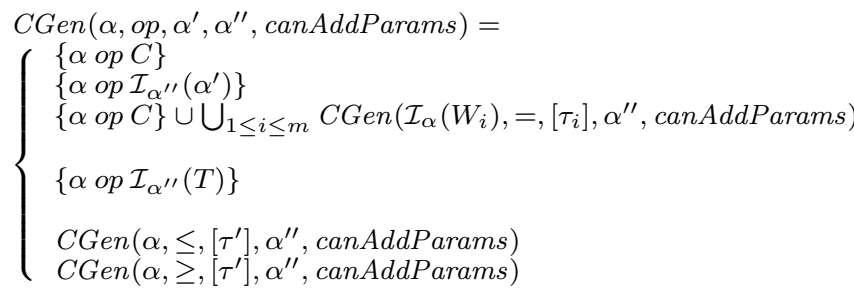

Figure 6. Auxiliary functions used by the constraint generation rules in Figure 5. Function CGen Wild is defined in Figure 11.

\begin{tabular}{|c|c|c|c|}
\hline \# & constraint & line & rules/cases \\
\hline$(\mathrm{x} 1)$ & $\mathcal{I}_{[c 4]}\left(\left[\right.\right.$ Cell.getC ret $\left.\left._{1}\right]\right) \leq[$ Wrapper.getW ret $]$ & 5 & $(\mathrm{r} 2),(\mathrm{r} 3), \mathrm{c} 2$ \\
\hline$(\mathrm{x} 2)$ & {$[\mathrm{t} 4] \leq \mathcal{I}_{[\mathrm{c} 4]}([\mathrm{t} 5])$} & 8 & $(\mathrm{r} 4), \mathrm{c} 2$ \\
\hline (x3) & {$[\mathrm{t} 5] \leq[$ data $]$} & 19 & $(\mathrm{r} 1)$ \\
\hline$(\mathrm{x} 4)$ & {$[$ data $] \leq\left[\right.$ Cell.get $\left.\mathrm{C}_{\text {ret }}\right]$} & 16 & $(\mathrm{r} 2)$ \\
\hline$(\mathrm{x} 5)$ & $\mathcal{I}_{[\mathrm{c} 5]}([$ Cell.getC ret $]) \leq[$ data $]$ & 22 & $(\mathrm{r} 1),(\mathrm{r} 3), \mathrm{c} 2$ \\
\hline (x6) & {$[$ data $] \leq \mathcal{I}_{[\operatorname{col}]}(E)$} & 25 & $(\mathrm{r} 4), \mathrm{c} 4$ \\
\hline$(\mathrm{x} 7)$ & data $]) \leq \mathcal{I}_{[\mathrm{c} 4]}([$ Cell.getC ret $])$ & closure & $(\mathrm{r} 7), \mathrm{x} 4$ \\
\hline$(\mathrm{x} 8)$ & $5]) \leq \mathcal{I}_{[\mathrm{c} 5]}([$ data $])$ & closur & $(\mathrm{r} 7$ \\
\hline$(\mathrm{x} 9)$ & data $]) \leq \mathcal{I}_{[\mathrm{c} 5]}\left(\left[\right.\right.$ Cell.getC ret $\left.\left._{\text {ret }}\right]\right)$ & closure & $(\mathrm{r} 7), \mathrm{x} 4$ \\
\hline$(\mathrm{x} 10)$ & $([\mathrm{t} 5]) \leq \mathcal{I}_{[\mathrm{c} 4]}([$ data $])$ & closure & $(\mathrm{r} 7), \mathrm{x} 3$ \\
\hline
\end{tabular}

Figure 7. Type constraints inferred from the program of Figure 2. Line numbers refer to Figure 2 and indicate from which program construct the constraint was generated. Rule numbers refer to generation rules in Figure 5, and case numbers refer to Figure 6.

In Figure 7, these are merged to form the (x1) constraint. Rows (x7)-(x10) in Figure 7 are the additional constraints that are generated due to the closure rules (r7) and (r8) of Figure 5.

\subsection{Constraint Solving}

A solution to the system of type constraints is computed using the iterative worklist algorithm of Figure 8. During solving, each variable $\alpha$ has an associated type estimate $\operatorname{Est}(\alpha)$. An estimate is a set of types, where types are as defined in Figure 4. Each estimate is initialized to the set of all possible (non-parametric) types and shrinks monotonically as the algorithm progresses. When the algorithm terminates, each estimate consists of exactly one type. Because type estimates do not contain parametric types, they are finite sets, and algebraic operations such as intersection can be performed directly. As an optimization, our implementation uses a symbolic representation for type estimates.

Algorithm Details. First, the algorithm initializes the type estimate for each constraint variable at lines 2 and 15-22 in Figure 8.

The algorithm uses a workset $P$ containing those constraint variables which it has decided shall become type parameters, but for which that decision has yet to be executed. The set $P$ is initially seeded with the constraint variable that corresponds to the declaration that is selected either by a heuristic or by the user (line 3 ). The inner loop of parameterize() (lines 5-11) repeatedly removes an element from $P$ and sets its estimate to a singleton type parameter. For new type parameters, the upper bound is the declared type in the original (unparameterized) program.

Whenever a type estimate changes, those changes must be propagated through the type constraints, possibly reducing the type estimates of other variables as well. The propagate() subroutine performs this operation, ensuring that the estimates on both sides of a type constraint contain only types that are consistent with the relation. Whenever a context variable $\mathcal{I}_{\alpha^{\prime}}(\alpha)$ gets resolved to a type parameter, $\alpha$ must also get resolved to type parameter (line 30 ). To see why, suppose that $\alpha$ gets resolved to a non-type parameter
Notation:

$\operatorname{Est}(\alpha) \quad$ a set of types, the type estimate of constraint variable $\alpha$ $\alpha_{\mathcal{P}} \quad$ type of constraint variable $\alpha$ in the original program $\operatorname{Sub}(\tau) \quad$ set of all non-wildcard subtypes of $\tau$

$\operatorname{Wild}(X)$ set of wildcard types (both lower- and upperbounded) for all types in type estimate $X$

$U_{E}^{S} \quad$ universe of all types, including all wildcard types (i.e., both super and extends wildcards)

1 Subroutine parameterize():

2 initialize()

$/ / P$ is a set of variables known to be type parameters

$3 P \longleftarrow$ automatically- or user-selected variable

4 repeat until all variables have single-type estimates

5 while $P$ is not empty do

$6 \quad \alpha_{t p} \longleftarrow$ remove element from $P$

$7 \quad$ if $\operatorname{Est}\left(\alpha_{t p}\right)$ contains a type parameter then

8

9

10

11

else $\operatorname{Est}\left(\alpha_{t p}\right) \longleftarrow$ type parameter from $\left.\operatorname{Est}\left(\alpha_{t p}\right)\right\}$ $\operatorname{Est}\left(\alpha_{t p}\right) \longleftarrow\{$ create new type parameter propagate ()

if $\exists \alpha .|\operatorname{Est}(\alpha)|>1$ then

$\operatorname{Est}(\alpha) \longleftarrow\{$ select a type from $\operatorname{Est}(\alpha)\}$ propagate()

// Set initial type estimate for each constraint variable

15 Subroutine initialize():

16 foreach non-context variable $\alpha$ do

$\mathbf{1 7}$ if $\alpha$ cannot have wildcard type then

$18 \operatorname{Est}(\alpha)=\operatorname{Sub}\left(\alpha_{\mathcal{P}}\right)$

19 else

$20 \operatorname{Est}(\alpha)=\operatorname{Sub}\left(\alpha_{\mathcal{P}}\right) \cup \operatorname{Wild}\left(\operatorname{Sub}\left(\alpha_{\mathcal{P}}\right)\right)$

21 foreach context variable $\mathcal{I}_{\alpha^{\prime}}(\alpha)$ do

$22 \operatorname{Est}\left(\mathcal{I}_{\alpha^{\prime}}(\alpha)\right)=U_{E}^{S}$

// Reconcile the left and right sides of each type inequality 23 Subroutine propagate():

24 repeat until fix-point (i.e., until estimates stop changing)

25 foreach constraint $\alpha \leq \alpha^{\prime}$ do

26 Remove from $\operatorname{Est}(\bar{\alpha})$ all types that are not a subtype of a type in $\operatorname{Est}\left(\alpha^{\prime}\right)$

27 Remove from $\operatorname{Est}\left(\alpha^{\prime}\right)$ all types that are not a supertype of a type in $\operatorname{Est}(\alpha)$

28 if $\operatorname{Est}(\alpha)$ or $\operatorname{Est}\left(\alpha^{\prime}\right)$ is empty then

29 stop: "No solution"

30 foreach context variable $\mathcal{I}_{\alpha^{\prime}}(\alpha)$ do

31 if $\operatorname{Est}\left(\mathcal{I}_{\alpha^{\prime}}(\alpha)\right)$ is a singleton set with type parameter $T$ and $\operatorname{Est}(\alpha)$ does not contain $T$ then add $\alpha$ to $P$

Figure 8: Pseudo-code for the constraint solving algorithm. 


\begin{tabular}{|c|c|c|c|c|c|c|c|c|c|}
\hline \multirow{2}{*}{$\begin{array}{l}\text { constraint } \\
\text { variable }\end{array}$} & \multirow[b]{2}{*}{ alias } & \multirow{2}{*}{$\begin{array}{c}\text { initial } \\
\text { estimate }\end{array}$} & \multicolumn{6}{|c|}{ intermediate solutions during solving } & \multirow{2}{*}{$\begin{array}{c}\text { inferred } \\
\text { type }\end{array}$} \\
\hline & & & (i) & (ii) & (iii) & (iv) & (v) & (vi,vii,viii) & \\
\hline c4 & $=\alpha_{1}$ & $\operatorname{Sub}(\mathrm{Cell})$ & $\longrightarrow$ & $\longrightarrow$ & $\longrightarrow$ & $\longrightarrow$ & $\longrightarrow$ & Cell $\}$ & Cell \\
\hline [Wrapper.getW $\left.W_{\text {ret }}\right]$ & $=\alpha_{2}$ & Sub(Object) & $\left\{\mathrm{T}_{1}\right\}$ & $\longrightarrow$ & $\longrightarrow$ & $\longrightarrow$ & $\longrightarrow$ & $\longrightarrow$ & $\mathrm{T}_{1}$ \\
\hline$[\mathrm{Cell}$.getC $\mathrm{ret}]$ & $=\alpha_{3}$ & Sub(object) & $\longrightarrow$ & $\left\{\right.$ Object, $\left.\mathrm{T}_{2}\right\}$ & $\left\{\mathrm{T}_{2}\right\}$ & $\longrightarrow$ & $\longrightarrow$ & $\longrightarrow$ & $\mathrm{T}_{2}$ \\
\hline [t5] & $=\alpha_{4}$ & Sub(Object) & $\longrightarrow$ & $\left\{\mathrm{T}_{2}\right\}$ & $\longrightarrow$ & $\longrightarrow$ & $\longrightarrow$ & $\longrightarrow$ & $\mathrm{T}_{2}$ \\
\hline [data] & $=\alpha_{5}$ & Sub(Object) & $\longrightarrow$ & $\left\{\right.$ Object, $\left.\mathrm{T}_{2}\right\}$ & $\left\{\mathrm{T}_{2}\right\}$ & $\longrightarrow$ & $\longrightarrow$ & $\longrightarrow$ & $\mathrm{T}_{2}$ \\
\hline $\mathcal{I}_{[\mathrm{c} 4]}([$ Cell.getC $\mathrm{ret}])$ & $=\alpha_{6}$ & $U_{E}^{S}$ & $\left\{\mathrm{~T}_{1}\right\}$ & $\longrightarrow$ & $\longrightarrow$ & $\longrightarrow$ & $\longrightarrow$ & $\longrightarrow$ & $\mathrm{T}_{1}$ \\
\hline $\mathcal{I}_{[c 4]}([$ data $])$ & $=\alpha_{7}$ & $U_{E}^{\stackrel{L}{S}}$ & $\left\{\mathrm{~T}_{1}\right\}$ & $\longrightarrow$ & $\longrightarrow$ & $\longrightarrow$ & $\longrightarrow$ & $\longrightarrow$ & $\mathrm{T}_{1}$ \\
\hline $\mathcal{I}_{[\mathrm{c} 4]}([\mathrm{t5}])$ & $=\alpha_{8}$ & $U_{E}^{\vec{S}}$ & $\left\{\mathrm{~T}_{1}\right\}$ & $\longrightarrow$ & $\longrightarrow$ & $\longrightarrow$ & $\longrightarrow$ & $\longrightarrow$ & $\mathrm{T}_{1}$ \\
\hline$[\mathrm{t} 4]$ & $=\alpha_{9}$ & Sub(object) & $\left\{\mathrm{T}_{1}\right\}$ & $\longrightarrow$ & $\longrightarrow$ & $\longrightarrow$ & $\longrightarrow$ & $\longrightarrow$ & $\mathrm{T}_{1}$ \\
\hline$[\mathrm{c5}]$ & $=\alpha_{10}$ & $\operatorname{Sub}(\mathrm{Cell})$ & $\longrightarrow$ & $\longrightarrow$ & $\longrightarrow$ & $\longrightarrow$ & $\longrightarrow$ & $\{\mathrm{Cell}\}$ & Cell \\
\hline $\mathcal{I}_{[\mathrm{c5}]}([\mathrm{t5}])$ & $=\alpha_{11}$ & $U_{E}^{S}$ & $\longrightarrow$ & $\longrightarrow$ & $\left\{\mathrm{T}_{2}\right.$, ? extends $\left.\mathrm{T}_{2}\right\}$ & $\longrightarrow$ & $\left\{\right.$ ? extends $\left.\mathrm{T}_{2}\right\}$ & $\longrightarrow$ & ? extends $\mathrm{T}_{2}$ \\
\hline $\mathcal{I}_{[\mathrm{c5}]}([\mathrm{Cell}$.getC ret $])$ & $=\alpha_{12}$ & $U_{E}^{\stackrel{E}{S}}$ & $\longrightarrow$ & $\longrightarrow$ & $\left\{\mathrm{T}_{2}\right.$, ? extends $\left.\mathrm{T}_{2}\right\}$ & $\longrightarrow$ & $\left\{\right.$ ? extends $\left.\mathrm{T}_{2}\right\}$ & $\longrightarrow$ & ? extends $\mathrm{T}_{2}$ \\
\hline $\mathcal{I}_{[\mathrm{c5}]}([$ data $])$ & $=\alpha_{13}$ & $U_{E}^{\stackrel{H}{S}}$ & $\longrightarrow$ & $\longrightarrow$ & $\left\{\mathrm{T}_{2}\right.$, ? extends $\left.\mathrm{T}_{2}\right\}$ & $\longrightarrow$ & $\left\{\right.$ ? extends $\left.\mathrm{T}_{2}\right\}$ & $\longrightarrow$ & ? extends $\mathrm{T}_{2}$ \\
\hline $\mathcal{I}_{[\operatorname{col}]}(E)$ & $=\alpha_{14}$ & $U_{E}^{\vec{S}}$ & $\longrightarrow$ & $X_{15}$ & $\left\{\mathrm{~T}_{2}\right.$, ? super $\left.\mathrm{T}_{2}\right\}$ & $\longrightarrow$ & $\longrightarrow$ & $\left\{\right.$ ? super $\left.\mathrm{T}_{2}\right\}$ & ? super $\mathrm{T}_{2}$ \\
\hline Workset $P$ & & $\left\{\alpha_{2}\right\}$ & $\alpha_{4}, \alpha_{3}, \alpha_{5}$ & $\left\{\alpha_{3}, \alpha_{5}\right\}$ & $\left\{\alpha_{5}\right\}$ & $\emptyset$ & $\emptyset$ & $\emptyset$ & $\emptyset$ \\
\hline
\end{tabular}

$X_{15}=\left\{\right.$ Object, ? super Object, $\mathrm{T}_{2}$, ? super $\left.\mathrm{T}_{2}\right\}$

Figure 9. Illustration of constraint solution for the program of Figure 2. Each constraint variable is given an alias for later reference. The table shows, for each constraint variable: its initial type estimate, its type estimate at 8 points in the solving process, and the final inferred type. A ' $\longrightarrow$ ' entry means that the type estimate for that constraint variable remained unchanged in the given step. Columns (i)-(iv) indicate the type estimates after each iteration of the loop on lines 5-11 of Figure 8 . Columns (v)-(viii) indicate the state after resolving under-constrained variables, on lines $12-14$ of Figure 8 . The last row shows the contents of the workset $P$, which contains variables that are eventually assigned type parameters.

type, $C$. In that case, the context is irrelevant (as mentioned in Section 3.1), and thus $\mathcal{I}_{\alpha^{\prime}}(\alpha)$ also must get resolved to $C$ (i.e., not a type parameter). This is a contradition. This step propagatates parameterization choices between classes.

Assembly of Parametric Types. The type estimates created during the constraint solution algorithm are all non-parametric, even for constraint variables that represent program entities whose type was parametric (e.g., c1 on line 12 in Figure 1), or will be parametric after refactoring (e.g., t1 on line 6 in Figure 1). A straightforward algorithm, run after solving, assembles these results into parametric types. For instance, the type Cell for [c5] and the type ? extends $\mathrm{T}_{2}$ for $\mathcal{I}_{[\mathrm{c} 5]}\left(T_{2}\right)$ are assembled into the type Cell<? extends $\mathrm{T}_{2}>$ for $[\mathrm{c} 5]$.

Example of Solving. Figure 9 illustrates the constraint solving process using the example program of Figure 2. The 'initial estimate' column in Figure 9 shows the initial type estimate for each constraint variable, determined as described in lines 15-22 of Figure 8 and using notation from the top of Figure 8. For example:

- The initial type estimate for variable [c4] is $S u b(\mathrm{Cell})$ because $\mathrm{Cell}$ is the original type of $c 4$ and $c 4$, being a variable declaration, cannot be assigned a wildcard type. The initial estimate is $\operatorname{Sub}(\mathrm{Cell})$, rather than $\{\mathrm{Cell}\}$, because the algorithm allows a newly introduced type parameter (e.g., $\mathrm{T}$ extends Cell, to be assigned to $c 4$ ).

- The initial estimate of $\mathcal{I}_{\text {[c5] }}([$ data] $])$ is $U_{E}^{S}$ (the universe of all types, including lower- and upper- bounded wildcards).

When the algorithm is run on the program of Figure 2, the outer loop (lines 4-14) is executed four times. On its first iteration, the inner loop (lines 5-11) is itself executed four times, and the inner loop is not executed on the subsequent iterations of the outer loop. Each inner loop execution consists of assigning a type parameter to a constraint variable. Each outer loop execution selects a type for an under-constrained constraint variable. We will now discuss columns (i)-(viii) of Figure 9, which show the state of the algorithm at the end of the four inner loop executions, and at the end of the four outer loop executions.

Column ( $i$ ). The algorithm first assigns a new type parameter $\mathrm{T}_{1}$ to constraint variable $\alpha_{2}$ (i.e., [Wrapper.getW $\mathrm{W}_{\text {ret }}$ ]), and propagates that choice to related constraint variables. Due to constraints (x1), $(\mathrm{x} 7),(\mathrm{x} 10)$, and $(\mathrm{x} 2)$, which establish the order $[\mathrm{t} 4] \leq \mathcal{I}_{[\mathrm{c} 4]}([\mathrm{t} 5])$ $\leq \mathcal{I}_{[\mathrm{c} 4]}([$ data $]) \leq \mathcal{I}_{[\mathrm{c} 4]}\left(\left[\right.\right.$ Cell. getC $\left.\left._{\text {ret }}\right]\right) \leq\left[\right.$ Wrapper $\cdot$ getW $\left._{\text {ret }}\right]$, the estimates of all 5 of these variables are narrowed to $\left\{\mathrm{T}_{1}\right\}$.
To understand why all 5 variables get the estimate $\left\{\mathrm{T}_{1}\right\}$, consider variable [t4]. Transitively, we have that: [t4] $\leq$ $\left[\right.$ Wrapper.getW $\left.W_{\text {ret }}\right]=\mathrm{T}_{1}$. The only subtypes of $\mathrm{T}_{1}$ are $\left\{\mathrm{T}_{1}\right.$, ? extends $\left.\mathrm{T}_{1}\right\}$. Intersecting this set with the current estimate for [t4] (namely, Sub(Object)) reduces [t4]'s estimate to simply $\mathrm{T}_{1}$. Propagating that change through the type constraints sets the other three constraint variables to $\mathrm{T}_{1}$ as well. Given that context variable $\mathcal{I}_{[\mathrm{c} 4]}([\mathrm{t5}])$ is now a type parameter, lines $30-32$ of the algorithm constrain t5 to be a type parameter as well (see the discussion of propagate() in Section 3.3). Similar arguments apply to $\mathcal{I}_{[\mathrm{c} 4]}([$ data $])$ and data, and to $\mathcal{I}_{[\mathrm{c} 4]}([$ Cell.getC $\mathrm{Cret}])$ and $\left[\right.$ Cell.getC ret $\left._{\text {, }}\right]$, so the three variables are added to the workset $P$ of variables that will be assigned type parameters. Column (i) shows the result.

Column (ii). The second variable to be removed from the workset is [t5] (an arbitrary choice is made at line 6 in the pseudocode); it is assigned a new type parameter $\mathrm{T}_{2}$. To satisfy the type constraints, the estimates of variables data, [Cell.getC $\mathrm{ret}_{\text {ret }}$, and $\mathcal{I}_{[\mathrm{col}]}(E)$ are narrowed, and column (ii) shows the result.

Column (iii). The third variable removed from the workset is $\left[\mathrm{Cell}\right.$.getC $\left.\mathrm{ret}_{\mathrm{ret}}\right]$. Its current estimate is $\left\{\right.$ Object, $\left.\mathrm{T}_{2}\right\}$. To assign a type parameter to the variable, line 8 of the algorithm chooses $\mathrm{T}_{2}$ (rather than creating a new type parameter). Because of constraints (x4), (x5), (x8), (x9), and (x6), the estimates of variables [data], $\mathcal{I}_{[\mathrm{c} 5]}\left(\left[\right.\right.$ Cell.getC $\left.\left.{ }_{\text {ret }}\right]\right), \mathcal{I}_{[\mathrm{c} 5]}([\mathrm{t} 5]), \mathcal{I}_{[\mathrm{c} 5]}([$ data $])$ and $\mathcal{I}_{[\mathrm{col}]}(E)$, respectively, must be narrowed. Column (iii) shows the result.

Column (iv). The algorithm removes the last variable, [data], from the workset. It is already assigned a type parameter, so its estimate remains unchanged. Estimates of other variables also remain unchanged in this iteration, because they are already consistent with the constraints. Column (iv) shows the result.

Column (v). At this point, the workset $P$ is empty and all estimates are consistent with the type constraints. However, the estimates for variables $\alpha_{1}$ and $\alpha_{10}-\alpha_{14}$ contain more than one type. The algorithm makes a heuristic choice (line 12 in pseudo-code) to narrow the estimate of $\mathcal{I}_{[\mathrm{c} 5]}\left(\left[\mathrm{Cell}\right.\right.$.getC $\left.\left.\mathrm{ret}_{1}\right]\right)$ to $\left\{\right.$ ? extends $\left.\mathrm{T}_{2}\right\}$. By reconciling that choice with constraints (x9) and (x8), the estimates for variables $\mathcal{I}_{[\mathrm{c} 5]}([$ data $])$ and $\mathcal{I}_{[\mathrm{c} 5]}([\mathrm{t5}])$, respectively, are also set to $\left\{\right.$ ? extends $\left.\mathrm{T}_{2}\right\}$. Column (v) shows the result.

Column (vi,vii,viii). The algorithm makes three more heuristic choices to narrow the estimates of $\mathcal{I}_{[\mathrm{col}]}(E),[\mathrm{c} 4]$, and [c5]. None of these choices causes any changes to propagate to other estimates. 
All type estimates are now singleton sets, and the solution shown in the rightmost column of Figure 9 is used for rewriting source code.

\subsubsection{Heuristics}

The algorithm of Figure 8 makes an underconstrained choice on lines 3,6,12, and 13. (On line 8, there is only one possibility.) Any choice yields a correct (behavior-preserving and type-safe) result, but some results are more useful to clients (e.g., permit elimination of more casts). Our implementation makes an arbitrary choice at lines 6 and 12, but uses heuristics at lines 3 and 13 to guide the algorithm to a useful result.

Type Uses for Parameterization. The selection of type uses for parameterization (on line 3) can affect the quality of the final parameterization. For example, in the example from Figure 1, selecting the parameter of MultiSet. contains (on line 18) would result in a parameterization in which too many type uses (including also the parameter of MultiSet.getCount) have the final type $T_{1}$. Such a (legal) parameterization would be inconvenient for clients of this class.

Our tool permits a user to select, with a mouse click, a type to parameterize. Otherwise, it uses the following heuristic.

1. If a generic supertype exists, use the supertype's signatures in the subtype. This is especially useful for customized container classes.

2. Parameterize the return value of a "retrieval" method. A retrieval method's result is downcasted by clients, or it has a name matching such strings as get and elementAt. Even classes that are not collections often have such retrieval methods [8].

3. Parameterize the formal parameter to an insertion method. An insertion method has a name matching such strings as add or put.

The heuristic further forbids selecting these uses of types:

1. Parameters of testing methods such as contains. Their types are typically different than the type parameter. For example, the parameter of java.util. Collection. contains is Object.

2. Type uses that are not in the public interface of the class.

3. Parameters of overridden methods (such as equals), unless their type in the overridden class is a type parameter. As discussed in Section 3.1, to preserve method overriding, types of such parameters must remain unchanged, and cannot be parameterized.

4. Type uses in interfaces or abstract classes. Their uses tend to be under-constrained and can lead to sub-optimal results.

Narrowing a Type Estimate to One Type. Given a type estimate to narrow, line 13 chooses one of its elements. The algorithm uses a heuristic that minimizes the use of casts in client code, while preserving flexibility in cases where this does not affect type-safety: it prefers (in this order):

i) types that preserve type erasure over those that do not,

ii) wildcard types over non-wildcard types, and

iii) type parameters over other types, but only if such a choice enables inference of type parameters for return types of methods.

For example, given estimate $\{$ Number, ? super Integer $\}$, if the type of the constraint variable in the original program was Number, the heuristic selects number. When selecting a type from estimate $\left\{T_{2}\right.$, ? super $\left.T_{2}\right\}$, the heuristic selects ? super $T_{2}$. Finally, when selecting from estimate $\left\{\right.$ Number, $\left.\mathrm{T}_{1}\right\}$, where $\mathrm{T}_{1}$ extends Number, the heuristic selects $\mathrm{T}_{1}$.

To justify the restriction in (iii), observe that assigning a type parameter or a parametric type to a method return type is beneficial, because doing so reduces the need for casts in clients of the class. Otherwise, introducing type parameters simply increases the apparent complexity of the class for clients. For example, consider variable $\circ 2$ on line 24 of Figure 1. Assigning a type parameter to o2 would not allow inferring a type parameter or a parametric type for any method's return type. Therefore, it is not assigned a type parameter but rather the type that preserves erasure (i.e., Object).

\subsubsection{Miscellaneous Issues}

A solution to the constraint problem does not always exist. For example, consider the following class.

$$
\text { class } C\{
$$$$
\text { \} }
$$

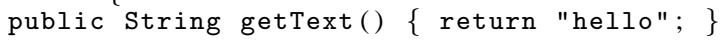

If the return type of getText is selected for parameterization, the type parameter would have to have a concrete lower bound: $\mathrm{T}$ super String. Such type parameters are disallowed in Java. Line 28 in Figure 8 detects cases in which no solution can be found.

As noted in Section 3.3, lines 30-32 of Figure 8 handle interclass dependencies. Interfaces and abstract classes are handled by the same mechanism, i.e., our algorithm creates type constraints to preserve method overriding and treats implements/extends relationships as other inter-class dependencies.

Our algorithm and implementation fully support parameterization in the presence of generic methods, e.g., those in java.util.Collections but we have not yet implemented adding type parameters to methods. (Von Dincklage and Diwan used heuristics to handle generic methods [22] — such heuristics may also be applicable to our work. In previous work, we used a contextsensitive version of the generic instantiation algorithm to parameterize methods [11].)

Reflection poses no problems for our algorithm-reflectionrelated classes are parameterized in JDK 1.5 and our inference uses those generic annotations when parameterizing user classes.

\subsection{Source Rewriting}

After the tool solves the constraint system and assigns each constraint variable a type, it rewrites the source code as follows:

1. Add a formal type parameter for each constraint variable whose type estimate is a type parameter. For example, a new formal type parameter $\mathrm{T}_{1}$ is added to class Wrapper on line 1 of Figure 2 because several constraint variables are assigned the type parameter $\mathrm{T}_{1}$.

2. Rewrite each program declaration to reflect the value of the corresponding constraint variable. For example, in Figure 9, the type assigned to $[\mathrm{t} 4]$ is $\mathrm{T}_{1}$. Therefore, on line 7 of Figure 2 , the declaration of $t 4$ is changed to $\mathrm{T}_{1}$.

3. Remove redundant casts, as on line 26 of Figure 1 . A cast $(C) E$ is considered redundant if the computed type for $[E]$ is $C$. If the computed type is a subtype of $C$, then the cast can be removed only if the cast expression is not used to force static member resolution. Otherwise the program's behavior will change [12].

\section{Evaluation}

A practical type parameterization tool must be correct, accurate, and usable. Correctness requires that run-time behavior is not modified for any client. Accuracy requires that the parameterization is close to what a human would have written by hand. Usability requires that the tool is easier to use than performing the refactoring manually. This section describes our experimental evaluation of these desiderata.

\subsection{Implementation}

We implemented our technique in a refactoring tool that is integrated with the Eclipse integrated development environment. Our previous work on the instantiation problem [11] was adopted by the Eclipse developers and made into Eclipse 3.1's INFER GENERIC TYPE ARGUMENTS refactoring. Our parameterization 


\begin{tabular}{|c|c|c|c|c|c|c|c|c|c|c|c|}
\hline \multirow[b]{2}{*}{ library } & \multicolumn{3}{|c|}{ parameterizable classes } & \multirow{2}{*}{$\begin{array}{l}\text { type use } \\
\text { selections }\end{array}$} & \multirow{2}{*}{$\begin{array}{l}\text { time } \\
\text { (sec.) }\end{array}$} & \multicolumn{3}{|c|}{ inference results } & \multicolumn{3}{|c|}{ diff vs. manual } \\
\hline & classes & LOC & type uses & & & params & wildcards & others & same & better & worse \\
\hline concurrent & 14 & 2715 & 415 & 5 & 115 & 11 & 23 & 266 & 353 & 37 & 25 \\
\hline apache & 74 & 9904 & 1183 & 53 & 301 & 70 & 90 & 676 & 1011 & 116 & 56 \\
\hline jutil & 9 & 305 & 80 & 4 & 1 & 7 & 5 & 40 & 65 & 15 & 0 \\
\hline jpaul & 17 & 827 & 178 & 11 & 6 & 15 & 11 & 110 & 148 & 22 & 8 \\
\hline amadeus & 8 & 604 & 129 & 4 & 5 & 6 & 4 & 86 & 125 & 1 & 3 \\
\hline dsa & 9 & 791 & 162 & 4 & 3 & 8 & 0 & 69 & 158 & 4 & 0 \\
\hline antlr & 10 & 601 & 140 & 3 & 6 & 12 & 8 & 81 & $\mathrm{n} / \mathrm{a}$ & $\mathrm{n} / \mathrm{a}$ & $\mathrm{n} / \mathrm{a}$ \\
\hline eclipse & 7 & 582 & 100 & 5 & 5 & 6 & 0 & 51 & $\mathrm{n} / \mathrm{a}$ & $\mathrm{n} / \mathrm{a}$ & $\mathrm{n} / \mathrm{a}$ \\
\hline Total & 148 & 16329 & 2387 & 89 & $\overline{442}$ & 135 & $\overline{141}$ & 1379 & 1860 & 195 & 92 \\
\hline
\end{tabular}

Figure 10. Experimental results. "Classes" is the number of parameterizable classes in the library, including their nested classes. "LOC" is lines of code. "Type uses" is the number of occurrences of a reference (non-primitive) type in the library; this is the maximal number of locations where a type parameter could be used instead. The next two columns show how many type use selections were made (line 3 of Figure 8) and the cumulative run time. After the results from our tool, the "diff vs. manual" columns indicate how our tool's output compares to the manual parameterization.

tool builds on that previous implementation work and is integrated with Eclipse in a similar way.

A programmer can use our tool interactively to direct a refactoring process (each step of which is automatic) by selecting (clicking on) an occurrence of a type in the program. The tool automatically rewrites (parameterizes) the class in which the mouse click occurred, and possibly other classes as well. Alternatively, a programmer can specify a set of classes to parameterize, and the tool heuristically selects type occurrences. The tool uses Eclipse's builtin support for displaying changes, and the user can examine them one-by-one, accept them, or back out of the changes.

\subsection{Methodology}

Our evaluation uses a combination of 6 libraries that have already been parameterized by their authors, and 2 libraries that have not yet been made generic; these two varieties of evaluation have complementary strengths and weaknesses. Use of alreadyparameterized libraries lets us evaluate our technique's accuracy by comparing it to the judgment of a human expert other than ourselves. However, it is possible that the library authors performed other refactorings at the same time as parameterization, to ease that task. Use of non-parameterized libraries avoids this potential problem, but the evaluation is more subjective, and a human reading the tool output may not notice as many problems as one who is performing the full task. (It would be easy for us to parameterize them ourselves, but such an approach has obvious methodological problems.)

Our experiments started with a complete, non-parameterized library. (For already-parameterized libraries, we first applied a tool that erased the formal and actual type parameters and added necessary type casts.) Not all classes are amenable to parameterization; we selected a subset of the library classes by first manually selecting the set of packages that we considered to be likely to contain parameterizable classes, and then selecting the classes that were parameterizable by our tool.

The experiments processed the classes of the library in the following order. We built a dependence graph of the classes, then applied our tool to each strongly connected component, starting with those classes that depended on no other (to-be-parameterized) classes. This is the same order a programmer faced with the problem would choose.

All experiments used our tool's fully automatic mode. For example, at each execution of line 3 of Figure 8, it chose the lexicographically first candidate type use, according to the heuristics of Section 3.3.1. To make the experiment objective and reproducible, we did not apply our own insight, nor did we rewrite source code to make it easier for our tool to handle, even when doing so would have improved the results.

Figure 10 lists the subject programs. All of these libraries were written by people other than the authors of this paper. concurrent is the java.util.concurrent package from Sun JDK 1.5. apache is the Apache collections library (larvalabs . com/collections/). jutil is a Java Utility Library (cscott.net/Projects/JUtil/). jpaul is the Java Program Analysis Utility Library (jpaul . sourceforge. net). amadeus is a data structure library (people.csail.mit.edu/ adonovan/). dsa is a collection of generic data structures (www . cs.fiu.edu/ weiss/\#dsaajava2). antlr is a parser generator (www . antlr.org). eclipse is a universal tooling platform (ww. eclipse. org). The last two libraries have not been parameterized by their authors.

Most of the classes are relatively small (the largest is 1303 lines), but this is true of Java classes in general. Our tool processes each class or related group of classes independently, so there is no obstacle to applying it to large programs.

\subsection{Results}

\subsubsection{Correctness}

A parameterization is correct if it is backward-compatible and selfconsistent. Backward compatibility requires that the erasure of the resulting parameterized classes is identical to the input. If so, then the compiled .class file behaves the same as the original, unparameterized version: for example, all method overriding relationships hold, exactly the same set of clients can be compiled and linked against it, etc. Consistency (type-correctness) requires that the parameterized classes satisfy the typing rules of Java generics; more specifically, that a Java compiler issues no errors when compiling the parameterized classes.

Our tool's output for all the tested library classes is correct: it is both backward-compatible and consistent.

\subsubsection{Accuracy}

We determined our tool's accuracy in different ways for libraries for which no no generic version is available (antlr and eclipse) and those for which a generic version is available (all others).

When no generic version of a library is available, we asked the developers their opinion of the result. A developer of Eclipse examined each of the changes made by our tool and concluded that the changes were "good and useful for code migration to Java 5.0." He mentioned only 1 instance (out of 100 uses of types in the Eclipse classes we parameterized) where the inferred result, while correct, could be improved. A developer of ANTLR concluded that the changes made by our tool "look pretty good". He mentioned 1 instance (out of 140 uses of types in the parameterized classes) where the inferred result, while correct, could be improved.

When a generic version of a library is available, we examined each difference between the pre-existing parameterization and our tool's output. For $87 \%$ of all type annotations, the output of our tool is identical or equally good. For $4 \%$ of annotations, the output of our tool is worse than that created by the human. For $9 \%$ of 
annotations, the tool output is better than that created by the human. Figure 10 tabulates the results.

Given two parameterizations, we used two criteria to decide which was better. The first, and more important, is which one allows more casts to be removed-in clients or inside the library itself. The secondary criterion is which one more closely follows the style used in the JDK collections, which were developed and refined over many years by a large group of experts, and which can therefore be reasonably considered models of style. The two criteria are in close agreement. (When multiple styles appear in the JDK, we did not count differences in either the "better" or "worse" category.) We present three examples from each category.

Examples when the output of our tool was worse:

i. Our tool does not instantiate the field next in member type LinkedBlockingQueue. Node (in concurrent) as Node $\langle\mathrm{E}\rangle$, but leaves it raw. Such a choice is safe, but it is less desirable than the manual parameterization.

ii. Our tool does not infer type parameters for methods; for example, the method PredicatedCollection. decorate in apache.

iii. Our tool inferred two separate type parameters for interface Buffer in the apache library. In this case the manual parameterization had only one.

Examples when the output of our tool was better (in each case, the developers of the package agreed the inferred solution was better than their manual parameterization):

i. Our tool adds a formal type parameter to member class Synchronousqueue. Node in concurrent. Adding the parameter allows elimination of several casts inside SynchronousQueue.

ii. In method VerboseWorkSet.containsAll in jpaul, our tool inferred an upper-bounded type parameter wildcard for the Collection parameter. This permits more flexible use and fewer casts by clients, and also adheres to the standard collections style from the JDK.

iii. Our tool inferred object as the type of the parameter of method Canonical.getIndex in amadeus. This is more flexible with fewer casts (and follows the JDK style). A similar case occurred in jpaul (our tool inferred object for the parameter of WorkSet. contains).

\subsubsection{Usability}

Our tool operated fully automatically, processing each class in under 3 seconds on average. A user who elected to manually select type uses would only need to make 89 mouse clicks to add 135 type parameters to 148 classes. As mentioned, $4 \%$ of the computed results are sub-optimal, requiring manual correction.

By comparison, manual parameterization requires making 1655 edits to add generic types - after reading the code to decide what edits to make. And even so, the human result was sub-optimal $9 \%$ of the time, so adjusting the results after finishing is even more work than in the tool-assisted case.

Those results illustrate that manual parameterization requires a significant amount of work. Parameterization of the apache collections took "a few weeks of programming", according to one of the developers. It is an error-prone activity and, to quote the same developer, "the main advantage we had was the over 11,000 test cases included with the project, that let us know we hadn't broken anything too badly."

\section{Related Work}

Duggan [9] presents an automatic approach for parameterizing classes written in PolyJava, a Java subset extended with parameterized types. Duggan's type inference infers one type parameter for each declaration in a class. Even after applying simplifications to reduce the number of useless type parameters, Duggan's analysis leads to classes with excess type parameters. Duggan's analysis is inapplicable to Java because PolyJava differs from Java 1.5 in several important ways, and Duggan does not address issues related to raw types, arrays of generic types, and wildcard types that arise in practice. Duggan does not report an implementation or empirical results.

Donovan and Ernst [7] present another automated approach for the parameterization of Java classes. The technique automatically determines both type parameters and where declarations should refer to those type parameters. The approach first performs an intraclass analysis that constructs a type constraint graph using dataflow rules. Then, after collapsing strongly connected components and making additional graph simplifications, an inter-class analysis fuses type parameters where required to preserve method overriding. The algorithm also determines how references to generic classes should be updated, by inferring actual type parameters. The work by Donovan and Ernst differs from ours in several significant ways. Although it is reported that the desired solution is computed for several examples, Donovan and Ernst report that "often the class is over-generalized" (has too many type parameters). Donovan and Ernst's work pre-dates Java 1.5 generics and targets a translation to GJ [3]. As a result, they may infer arrays of generic types (disallowed in Java 1.5 generics), and do not consider the inference of wildcard types. Donovan's work [7] was never fully implemented, and no empirical results are reported.

Von Dincklage and Diwan [22] also present a combined approach for the parameterization of classes and for the inference of actual type parameters in clients of those classes. Similarly to Duggan [9], their tool (Ilwith) creates one type parameter per declaration, then uses heuristics to merge type parameters. Our system differs in its (1) algorithm, (2) implementation, and (3) evaluation. (1) A key difference from our algorithm is that Ilwith is unsound, due to insufficient type constraints (it is missing those for preserving erasure for methods and fields, and overriding relationships between methods). As a result, the behavior of both the library and its clients may change after parameterization, without warning; this makes the technique unsuitable in practice. By contrast, our approach is correct (see Section 4.3.1) and uses heuristics only to choose among legal solutions. Unlike our approach, Ilwith does not handle key features of Java generics such as raw types and wildcards. To control run time and the number of constraint variables, Ilwith uses special cases in the algorithm to handle other Java features, such as calls to static methods and methods in generic classes, and context-, field-, and instance-sensitivity; by contrast, our system is more uniform, and we have not found performance to be a problem. Ilwith creates maximally many type parameters and then tries to merge them via heuristics (though other heuristics, such as the requirement that every field declaration mentions a type parameter, may leave the result over-general). By contrast, our technique starts with no type parameters and incrementally adds them. (2) We mention only two differences between the two implementations. First, Ilwith does not rewrite source code, but merely prints method signatures without providing details on how method bodies should be transformed. Second, Ilwith took "less than 2 minutes" per class on a $2.66 \mathrm{GHz}$ machine, whereas our implementation averaged less than 3 seconds per class on a $2.2 \mathrm{GHz}$ machine. (3) The experimental evaluation of the two tools differs as well. Ilwith was evaluated on 9 data structures ( 5 lists, 1 stack, 1 set, and 2 maps) chosen from two libraries (47 classes altogether, including inner classes, interfaces and abstract classes). The authors made whatever edits were necessary to enable Ilwith to succeed, so the classes are most like the pre-parameterized libraries in our evaluation. However, the authors did not evaluate the accuracy of the solution, either via examination by a Java expert or via comparison to existing parameterized versions of the libraries (then available, for example, from the GJ project and from JDK 1.5 beta releases). Even the example signatures shown in the paper differ from what a programmer would have written manually, for example in addAll, 
contains, equals, putAll, remove, and removeEntryForKey. (The authors state that the results are consistent with Eiffel [14], but a Java programmer performing a refactoring is more likely to care about Java semantics and backward compatibility.)

Previous work by the present authors includes two algorithms [8, 11] that, given a set of generic classes, infer how client code can be updated by inferring actual type parameters and removing casts that have been rendered redundant. In this paper, we extended the constraint formalism and implementation of [11] to accommodate the inference of wildcard types. Although [11] includes a mode for inferring method type parameters by means of a context-sensitive analysis, it does not infer class type parameters. Some of the present authors previously used type constraints for several refactorings and program transformations that also involve manipulating types and class hierarchies, such as refactorings related to generalization [21], customization of library classes [6], and refactorings for migration applications between similar library classes [1]. The INFER TYPE refactoring by Steimann et al. [20] lets a programmer select a given variable and determines a minimal interface that can be used as the type for that variable. If such an interface does not yet exist, it is created automatically. Steimann et al. only present their type inference algorithm informally, but they appear to use similar constraints to those of [21].

A number of authors have explored compile-time parametric type inference to ease the burden of explicit parameterization in languages supporting parametric types [15, 18, 13]. Many of these approaches were applied to functional programming languages, and thus focus on introducing type parameters for functions, rather than for classes or modules. Obviously, such languages differ in many important respects from Java 5.0, e.g. in the lack of a class hierarchy with inheritance and overriding, or in the use of structural (cf. nominal) subtyping, or in the lack of the notion of type erasure. These differences in semantics and structure necessitate significantly different constraint systems. Moreover, the type systems in many functional languages (e.g. ML) induce a unique principle type for each program variable, whereas in our case the constraint system leaves the possibility to select a "desirable" result from a software engineering perspective, which is a critical concern for source-to-source transformations. Yet other works describe parametric type inference even for languages with mandatory explicit parameterization for the purpose of statically type checking such diverse languages as Cecil [4], constraint logic programming (CLP) [10] or ML [16]. Again, these works differ from ours in many critical details of the language's type system.

Siff and Reps [19] focused on translating C functions into C++ function templates by using type inference to detect latent polymorphism. In their work, opportunities for introducing polymorphism stem from operator overloading, references to constants that can be wrapped by constructor calls, and from structure subtyping. In a similar vein, De Sutter et al. [5] perform link-time inference of reusable $\mathrm{C}++$ object code fragments for the purpose of code compaction. In essence, their work reconstitutes type-parametric functions from template instantiations created during compilation, but does so using code similarity detection rather than type inference per se, and on a low level representation (object code).

\section{Conclusion}

We have presented a solution to the parameterization problem, which involves adding type parameters to existing, non-generic class declarations. This is a complex task due to requirements of backward compatibility and behavior preservation, the existence of multiple type-correct solutions, complications posed by raw and wildcard types, and the necessity to simultaneously parameterize and (generically) instantiate multiple interrelated classes. Our algorithm handles all these issues and the full Java language.

Our parameterization algorithm subsumes previous algorithms for generic instantiation, which change library clients to take ad-

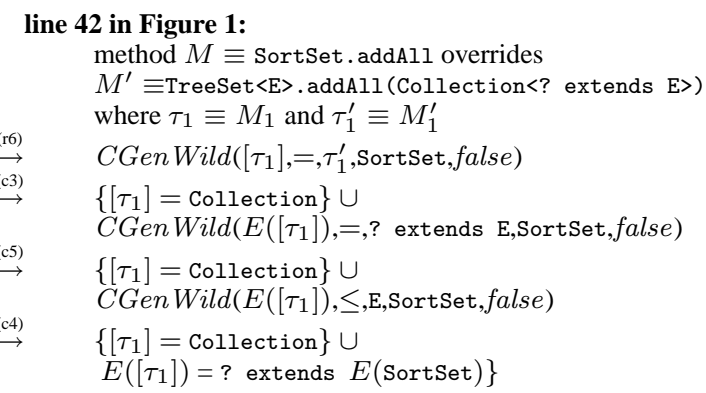

Figure 12. Example applications of constraint generation rules from Figure 5.

vantage of libraries that have been made generic. Our analysis computes an instantiation at the same time as it performs parameterization.

We have implemented our algorithm in the context of the Eclipse IDE and run experiments to verify its correctness, accuracy, and usability. The results are correct: they are backward-compatible and they maintain behavior. The results are even more accurate than parameterizations performed by the library authors: $9 \%$ of the tool results are better, and $4 \%$ of the tool results are worse. The tool is usable: it requires only a mouse click, runs quickly, and thus greatly reduces overall developer effort. We are hopeful that this tool may increase the uptake of Java generics, which are a very valuable language feature that increases type safety and expressiveness.

\section{References}

[1] I. Balaban, F. Tip, and R. Fuhrer. Refactoring support for class library migration. In OOPSLA, pages 265-279, Oct. 2005.

[2] G. Bracha, 2005. Personal communication.

[3] G. Bracha, M. Odersky, D. Stoutamire, and P. Wadler. Making the future safe for the past: Adding genericity to the Java programming language. In OOPSLA, pages 183-200, Oct. 1998.

[4] C. Chambers. The Cecil language specification and rationale: Version 2.0. 1996.

[5] B. De Sutter, B. De Bus, and K. De Bosschere. Sifting out the mud: low level c++ code reuse. In OOPSLA, pages 275-291, Oct. 2002.

[6] B. De Sutter, F. Tip, and J. Dolby. Customization of Java library classes using type constraints and profile information. In ECOOP, pages 585-610, June 2004.

[7] A. Donovan and M. Ernst. Inference of generic types in Java. Technical Report MIT/LCS/TR-889, MIT, Mar. 2003.

[8] A. Donovan, A. Kieżun, M. S. Tschantz, and M. D. Ernst. Converting Java programs to use generic libraries. In OOPSLA, pages 15-34, Oct. 2004.

[9] D. Duggan. Modular type-based reverse engineering of parameterized types in Java code. In OOPSLA, pages 97113, Nov. 1999.

[10] F. Fages and E. Coquery. Typing constraint logic programs. Theory and Practice of Logic Programming, 1(6):751-777, 2001.

[11] R. Fuhrer, F. Tip, A. Kieżun, J. Dolby, and M. Keller. Efficiently refactoring Java applications to use generic libraries. In ECOOP, pages 71-96, July 2005.

[12] J. Gosling, B. Joy, G. Steele, and G. Bracha. The Java Language Specification. Addison Wesley, Boston, MA, third edition, 2005.

[13] S. P. Jones, D. Vytiniotis, S. Weirich, and G. Washburn. Simple unification-based type inference for GADTs. In ICFP, 
One returns a singleton set whose only constraint relates type constraint variables $\alpha$ and $\alpha^{\prime}$ according to op

$$
\begin{aligned}
& \text { One }\left(\alpha, o p, \alpha^{\prime}\right)= \\
& \begin{cases}\left\{\alpha \text { op } \alpha^{\prime}\right\} & \text { when } o p \equiv= \\
\left\{\alpha=? \text { extends } \alpha^{\prime}\right\} & \text { when } o p \equiv \leq \\
\left\{\alpha=? \text { super } \alpha^{\prime}\right\} & \text { when } o p \equiv \geq\end{cases}
\end{aligned}
$$

CGenWild is like $C G e n$, but may generate wildcard constraints.
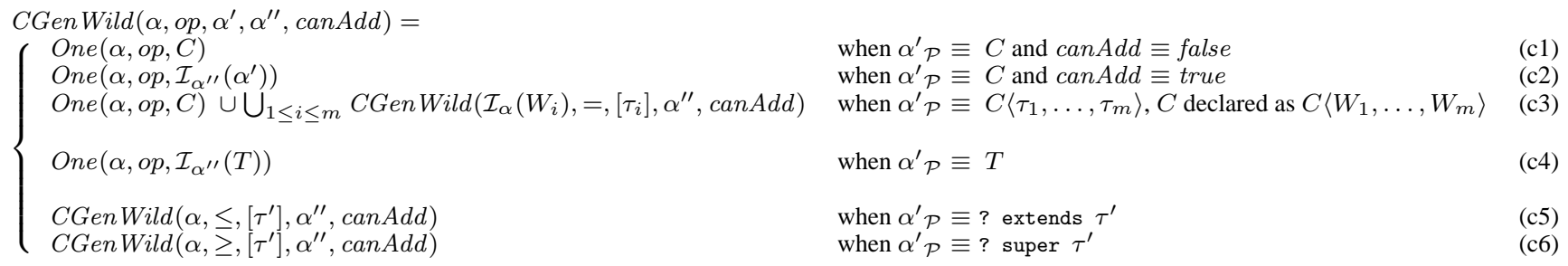

Figure 11. CGen Wild auxiliary function, which is used in Figure 5. CGen Wild is like function CGen of Figure 6, except for the use of function One.

Sept. 2006.

[14] B. Meyer. Object-Oriented Software Construction. Prentice Hall, second edition, 1997.

[15] R. Milner, M. Tofte, R. Harper, and D. MacQueen. The Definition of Standard ML - Revised. MIT Press, 1997.

[16] A. Ohori and P. Buneman. Static type inference for parametric classes. In OOPSLA, pages 445-456, Oct. 1989.

[17] J. Palsberg and M. Schwartzbach. Object-Oriented Type Systems. John Wiley \& Sons, 1993.

[18] A. Rodriguez, J. Jeuring, and A. Loh. Type inference for generic Haskell. Technical Report UU-CS-2005-060, Utrecht Univ., 2005.

[19] M. Siff and T. Reps. Program generalization for software reuse: From C to C++. In FSE, pages 135-146, Oct. 1996.

[20] F. Steimann, P. Mayer, and A. Meißner. Decoupling classes with inferred interfaces. In SAC, pages 1404-1408, Apr. 2006.

[21] F. Tip, A. Kieżun, and D. Bäumer. Refactoring for generalization using type constraints. In OOPSLA, pages 13-26, Nov. 2003.

[22] D. von Dincklage and A. Diwan. Converting Java classes to use generics. In OOPSLA, pages 1-14, Oct. 2004. 


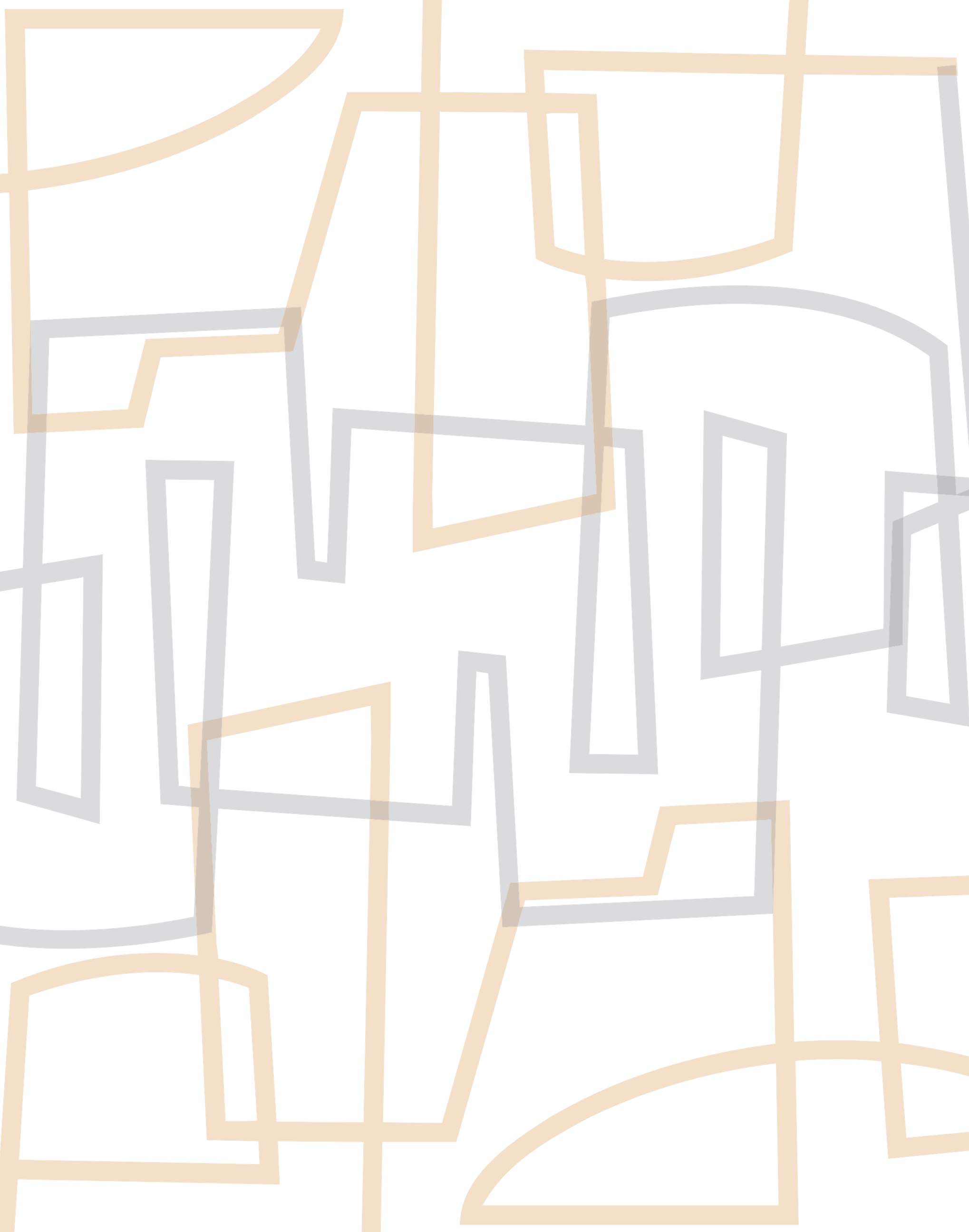

